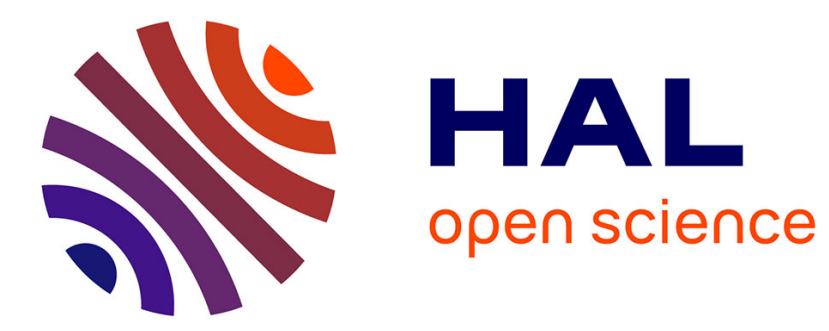

\title{
Functional Monochalcogenides: Raman Evidence Linking Properties, Structure, and Metavalent Bonding
} Christophe Bellin, Amit Pawbake, Lorenzo Paulatto, Keevin Béneut, Johan

Biscaras, Chandrabhas Narayana, Alain Polian, Dattatray J. Late, Abhay Shukla

\section{To cite this version:}

Christophe Bellin, Amit Pawbake, Lorenzo Paulatto, Keevin Béneut, Johan Biscaras, et al.. Functional Monochalcogenides: Raman Evidence Linking Properties, Structure, and Metavalent Bonding. Physical Review Letters, 2020, 125 (14), pp.145301. 10.1103/PhysRevLett.125.145301 . hal-02969547

\section{HAL Id: hal-02969547 \\ https://hal.sorbonne-universite.fr/hal-02969547}

Submitted on 16 Oct 2020

HAL is a multi-disciplinary open access archive for the deposit and dissemination of scientific research documents, whether they are published or not. The documents may come from teaching and research institutions in France or abroad, or from public or private research centers.
L'archive ouverte pluridisciplinaire HAL, est destinée au dépôt et à la diffusion de documents scientifiques de niveau recherche, publiés ou non, émanant des établissements d'enseignement et de recherche français ou étrangers, des laboratoires publics ou privés. 


\title{
Functional Monochalcogenides: Raman Evidence Linking Properties, Structure and Metavalent Bonding
}

Christophe Bellin, ${ }^{1, *}$ Amit Pawbake,,${ }^{1,2, *}$ Lorenzo Paulatto, ${ }^{1}$ Keevin Béneut,${ }^{1}$ Johan Biscaras, ${ }^{1}$ Chandrabhas Narayana, ${ }^{3}$ Alain Polian, ${ }^{1}$ Dattatray J. Late, ${ }^{4}$ and Abhay Shukla ${ }^{1, \dagger}$

${ }^{1}$ Institut de Minéralogie, de Physique des Matériaux et de Cosmochimie, Sorbonne Université, UMR CNRS 7590, MNHN, IRD UMR 206, 4 Place Jussieu, F-75005 Paris, France

${ }^{2}$ Institut Neel CNRS/UGA UPR2940, MCBT, 25 rue des Martyrs BP 166, 38042, Grenoble cedex 9, France

${ }^{3}$ Chemistry and Physics of Materials Unit, Jawaharlal Nehru Centre for Advanced Scientific

Research, Jakkur, Bangalore 560 064, India

${ }^{4}$ Centre for Nanoscience and Nanotechnology Amity University Maharashtra, Mumbai- Pune Expressway, Bhatan, Post Somatne, Panvel, Mumbai, Maharashtra 410206, India

\begin{abstract}
Pressure and temperature dependent Raman scattering in GeSe, SnSe and GeTe for pressures beyond $50 \mathrm{GPa}$ and for temperatures ranging from $78 \mathrm{~K}$ to $800 \mathrm{~K}$ allow us to identify structural and electronic phase transitions, similarities between GeSe and SnSe and differences with GeTe. Calculations help to deduce the propensity of GeTe for defect formation and the doping that results from it, which gives rise to strong Raman damping beyond anomalous anharmonicity. These properties are related to the underlying chemical bonding and consistent with a recent classification of bonding in several chalcogenide materials which puts GeTe in a separate class of 'incipient' metals.
\end{abstract}

DOI:

PACS numbers: 
Recent work aiming to classify chalcogenides on the basis of several calculated or measured properties has led to the conclusion that anomalous behaviour in potential materials for thermoelectric or phase change applications can be largely ascribed to a specific type of chemical bonding.

The link to a specific 'resonant' bonding mechanism was first suggested by Lucovsky and White for GeTe[1], generalized and refined in subsequent works with respect to dielectric properties[2], bonding [3] and thermal properties [4]. Using coordination number, anharmonicity, bond polarizability and conductivity to classify materials, this 'metavalent' bonding [5, 6] was identified as different from the classical 'resonant' bonding and is now thought to be emblematic for certain properties like transport or optical contrast in these materials $[7,8]$, defined by the competition between electronic localization in insulating ionic or covalent solids and delocalization in conducting metals. Phenomena like anharmonicity on the other hand [5] are exacerbated and should show up in pressure and temperature dependence of phonon modes.

Here we provide spectroscopic evidence in the form of pressure and temperature dependent Raman measurements in GeSe, SnSe and GeTe. In these IV-VI materials, the column IV and column VI elements are neighbours and the resulting binary compounds form a good basis for comparison of properties. The prototype phase change material GeTe is rhombohedral in ambient conditions and transits to a cubic $\mathrm{NaCl}$ structure and then to an orthorhombic structure as pressure increases [9]. A metastable cubic $\mathrm{NaCl}$ phase is also found in ambient conditions. GeSe and SnSe are known to undergo orthorhombic to orthorhombic transitions at low pressure as well as semiconductor to metal transitions. SnSe meanwhile is claimed to be a promising thermoelectric material at high temperature [10]. We compare pressure dependent Raman spectra in SnSe and GeSe and find them to be remarkably similar. Their behaviour is markedly different from our earlier findings in GeTe [9]. We also compare temperature dependent Raman spectra between GeSe and GeTe and find a strong contrast. GeSe shows standard temperature dependent softening and broadening and no phase change. GeTe shows anomalous softening and broadening and a high temperature phase change all of which can be linked to the formation of Ge vacancies and ultimately to the unique bonding mechanism in this material.

Much of past work in these materials has been accomplished using X-ray and neutron diffraction. Raman spectroscopy is complementary to structural studies and sensitive to 
aspects like anharmonicity, electron phonon coupling and iso-structural or subtle phase changes as our recent study on the pressure phase diagram of GeTe has shown [9]. A study of the ambient pressure phases of GeSe found an orthorhombic to cubic phase transition at $651{ }^{\circ} \mathrm{C}$, just below the melting temperature of $670{ }^{\circ} \mathrm{C}$ [11]. Pressure studies confirm the persistence of the orthorhombic phase [12] or find a continuous Pmcn to Ccmm transition [13], notably accompanied by a sharp drop in resistance between 20 and 25 GPa.

SnSe at ambient pressure undergoes a transition within the orthorhombic structure from Pnma to Cmcm complete at about 800 K [14-16]. Most high pressure studies indicate similar subtle transitions. Indeed the same transition is found at about $10 \mathrm{GPa}$ by high pressure X-ray diffraction and Raman studies [16, 17]. Another study [18] finds this transition at around $15 \mathrm{GPa}$ with the appearance of a cubic phase at $27 \mathrm{GPa}$ in a gradual evolution which is complete only beyond $45 \mathrm{GPa}$. Calculations [19, 20] find the Pnma to Cmcm transition at low pressure $(2.5 \mathrm{GPa}-6 \mathrm{GPa})$ and a transition to the cubic phase at high pressures nearing $40 \mathrm{GPa}$ while a lone study reported a transition to an unusual P21/c structure at $12.6 \mathrm{GPa}[21]$.

All monochalcogenide single crystals were purchased from 2D Semiconductors (USA). For high pressure measurements a freshly cleaved flake was loaded in a membrane diamond anvil cell(DAC) [22] using a Rhenium gasket with a $250 \mu \mathrm{m}$ diamond culet and Neon as the pressure transmitting medium [23]. Neon ensures quasi-hydrostatic conditions, has no Raman activity and is chemically inert avoiding surface contamination in the pressure cell. The R1-line emission of a ruby [24] was used for pressure calibration [25]. A Jobin-Yvon HR460 spectrometer (1500 grooves/mm monochromator and Andor CCD camera, spectrometer resolution being $1.5 \mathrm{~cm}^{-1}$ ) in backscattering geometry was used for the Raman scattering experiments.

The primary beam was from a $514.5 \mathrm{~nm}$ Ar laser focused into a $2 \mu \mathrm{m}$ spot. Incident power on the DAC was limited below $40 \mathrm{~mW}$ (and $120 \mathrm{~mW}$ for high pressure phases). The $12 \mathrm{~cm}^{-1}$ low frequency cut-off, crucial for many low frequency peaks, was achieved with Rayleigh rejection using three volume Bragg filters. A remnant low energy tail was subtracted using a polynomial background. For temperature dependent Raman spectra the sample was placed in a water-cooled Linkam TS1500 stage in a flow of pure Argon. The ambient and high pressure data were recorded in the range of 12 to $1200 \mathrm{~cm}^{-1}$ and acquired for 120 to 900 seconds per pressure point depending on count rate. 
All calculations were performed using the Quantum-ESPRESSO suite of codes[26, 27] including phonons and Raman cross sections from perturbation theory[28, 29] and anharmonic properties using D3Q codes[30]. For all the simulations we used norm-conserving pseudopotentials from the SG15-ONCV library[31, 32] and the PBE gradient-corrected local functional with van der Waals Grimme-D2 correction.[33, 34] The Fourier transform grid was kept constant at different pressures to ensure consistency. In the worst case (larger cell) it corresponds to a kinetic energy cutoff of at least 60 Ry. For sampling the electronic reciprocal space we used a $4 \times 4 \times 4$ grid for the $\mathrm{Cmcm}$ structure and 4 points along the short cell directions and 2 points along the long ones for the Pcmn and Pnma structures. For the phonon-phonon interaction calculation in GeTe we computed the 2nd and 3rd dynamical matrices over $8 \times 8 \times 8$ and $4 \times 4 \times 4$ grid points respectively. In order to integrate the spectral weight equation[35, 36], the matrices were then converted to 2 and 3-body force constants and Fourier-interpolated over a grid of $70 \times 70 \times 70$ points.

Ambient pressure and temperature phases of bulk GeSe and SnSe are orthorhombic with eight atoms per unit cell [37] and 12 Raman active modes $\left(4 A_{g}+2 B_{1 g}+4 B_{2 g}+2 B_{3 g}\right)$. The $4 B_{2 g}$ modes are difficult to detect due to a weak Raman tensor but the $B_{1 g}$ modes (GeSe) and the $B_{3 g}$ modes (SnSe) can be observed for unpolarized Raman backscattering spectra along the c axis, as seen in the lowest pressure spectra of Figure 1(a) and Figure 1(c) which are in good agreement with previous reports at ambient conditions [38-40]. The modes are symmetry indexed in Figure 1(b) and Figure 1(d). In many previous reports low energy phonons remained undetected, often due to instrumental reasons [39-41].

As can be seen in Figure 1 the pressure variation is remarkably similar in GeSe and SnSe. It is however markedly different for GeTe, as reported in our recent work [9]. The Raman signal vanishes with pressure in both GeSe and SnSe, beyond approximately 35 GPa in GeSe and beyond $10 \mathrm{GPa}$ in SnSe, though a couple of modes survive to about $50 \mathrm{GPa} . A_{g}{ }^{1}$, $A_{g}{ }^{3}, B_{1 g}$ and $B_{3 g}$ modes in both materials behave anomalously, with little dispersion, first hardening and then softening as pressure increases. $A_{g}{ }^{2}, A_{g}{ }^{4}$ modes show a monotonous hardening. The vanishing Raman signal is probably due to strong damping from increasing carrier concentration and electronic transition to a small gap semi conductor or a semi-metal. The ambient condition band gap is $2.29 \mathrm{eV}$ in GeSe and $1.79 \mathrm{eV}$ in SnSe. The Raman signal could also disappear due to a phase transition to a cubic phase though this seems to be excluded from earlier diffraction work for GeSe $[12,13]$. As detailed above, in GeSe some 

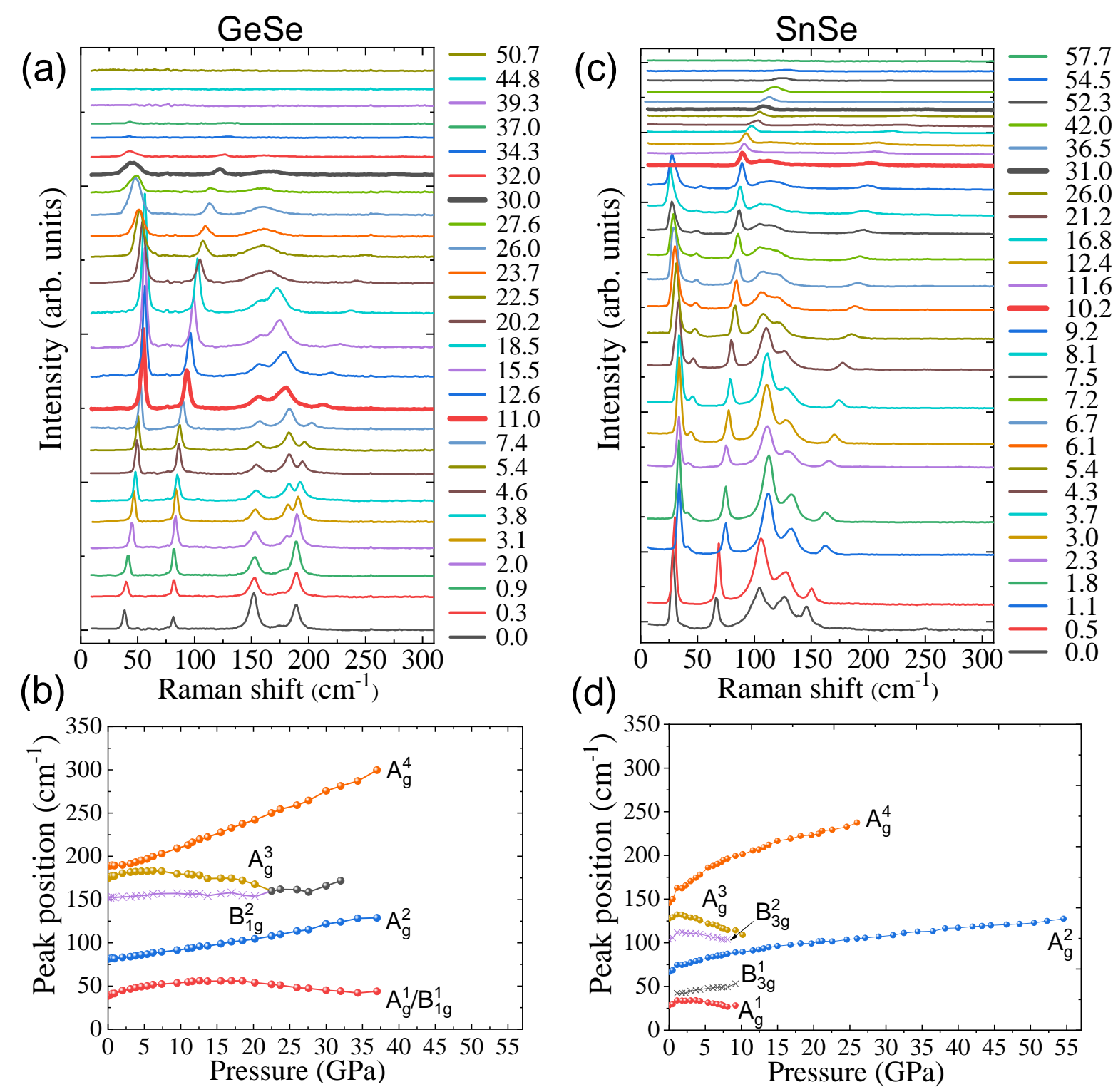

FIG. 1. Pressure dependent Raman spectra of (a) GeSe and (b) SnSe, showing the pressure dependence of $A_{g}$ modes (dots) and $B_{1 g}$ and $B_{3 g}$ modes (crosses). Pressure increases from bottom to top and values are in GPa. The Raman spectra around 10 and $30 \mathrm{GPa}$ are shown with a thick line to enable easy comparison between the two materials. Extracted Raman shifts and their pressure variation for (c) GeSe and (d) SnSe show the overall similarity of the two materials.

diffraction studies do evoke a structural transition from the Pmcn to the Cmcm space group [13] while others [12] do not. From our Raman data we cannot detect any such structural change. Ab-initio calculations indicate that the Pnma and the higher symmetry Cmcm structures are very similar but do rule out the possibility of the trigonal structure which 
only has two active modes (see Supplemental material). Similarly in SnSe, an experimental X-ray diffraction study finds a gradual transition from ambient pressure Pnma to Cmcm, complete at $10.5 \mathrm{GPa}[17]$.

Since only two weak Raman lines persist above this pressure in our data, and Cmcm is a structure with higher symmetry, our data is compatible with this scenario. A cubic $\mathrm{CsCl}$ phase has been reported in SnSe at a pressure of $27 \mathrm{GPa}$ [18] with the transition completed at $45 \mathrm{GPa}$, consistent with some simulations [19,20] and with the disappearing Raman signal at these high pressures in our work. From ab-initio simulation, the Cmcm phase becomes increasingly symmetric with pressure. However calculated Raman cross sections can vary enormously as mentioned earlier and a further complication arises because the system is metallic above $10 \mathrm{GPa}$ at the DFT local-density approximation level.

Beyond these similarities, some differences exist. In GeSe, though the Raman signal completely disappears only beyond $35 \mathrm{GPa}$, there is an abrupt drop in the intensity of the Raman lines and a corresponding broadening at a pressure of $20 \mathrm{GPa}$, signifying increased damping (see Supplemental material). Interestingly transport measurements as a function of pressure in GeSe show that though the resistance drops continuously with pressure over several orders of magnitude, there is an abrupt drop between 20 and $25 \mathrm{GPa}[12,13]$, signifying a change in electronic structure. The increased damping of the Raman lines could be due to an increase in charge carriers as seen in several experimental and theoretical studies of doping in semiconductors [42-45].

In SnSe we detect a discontinuous change in Raman frequencies of all modes with a jump of $12 \%$ at $1.1 \mathrm{GPa}$. Though theoretical studies evoke a low pressure structural change (Pnma to Cmcm, $[19,20])$ the Raman spectrum remains identical indicating an isostructural transition at this low pressure. In summary, in GeSe, the structure remains orthorhombic Pnma but pressure induces metallicity and an abrupt damping of Raman peaks is observed at $20 \mathrm{GPa}$ with complete disappearance of the Raman signal between 35 and $40 \mathrm{GPa}$. In SnSe, an isostructural transition within the Pnma structure is seen at 1.1 GPa. At $10 \mathrm{GPa}$ a transition to the Cmcm structure is probable. Between $27 \mathrm{GPa}$ and $55 \mathrm{GPa}$ the single remnant Raman peak disappears, which is interpreted as a transition to a cubic structure with accompanying metallicity [18].

Finally we come back to the observation (Figure 1) that $A_{g}{ }^{1}, A_{g}{ }^{3}, B_{1 g}$ and $B_{3 g}$ modes in both GeSe and SnSe disperse little, with visible softening for some modes as pressure 
increases. Softening is accompanied by line broadening and damping (see Supplemental material) and as we have pointed out above, these can be explained by an increasing metallic nature of the materials. These observations are compatible with a change in the prevalently covalent bonding of these materials towards metavalent bonding. In effect both materials should increasingly move towards an incipient metallic state with increasing pressure because pressure induced electron delocalization reduces both electron transfer (ionicity) and sharing (covalency). Furthermore, as observed experimentally, this should happen at lower pressures for SnSe than for GeSe which is more covalent and has a lower conductivity in ambient conditions $[5,6]$.

The dependence on temperature for SnSe can be consulted in several recent studies (for example [40]). Clearly all modes in SnSe broaden with temperature testifying to strong anharmonicity. In SnSe an orthorhombic Pnma to Cmcm transition takes place around 800 $\mathrm{K}$ with the disappearance of some modes in the more symmetric Cmcm structure which is however not Raman silent because the $A_{g}{ }^{2}$ mode persists. In Figure 2 we show the temperature variation of the Raman signal for GeTe (Figure 2a) and GeSe (Figure 2b). GeSe shows standard behaviour as Raman modes soften marginally and broaden with temperature in keeping with lattice expansion and increasing anharmonicity. No change in crystal structure can be seen and none is expected since the transition to the cubic phase occurs at higher temperature. GeTe on the other hand shows anomalous broadening of its two modes even at low temperature. The broadening increases considerably beyond $300 \mathrm{~K}$ and beyond $550 \mathrm{~K}$ the two distinct maxima are lost in a broad and very low intensity background which shifts to higher energy between $600 \mathrm{~K}$ and $800 \mathrm{~K}$.

It must be remembered that beyond $700 \mathrm{~K}$ a transition to the Raman silent cubic structure takes place. The $300 \mathrm{~K}$ measurement before heating (black line) and after cooling down (green line) are quite similar, showing that the high temperature phase transition is reversible. However small differences can be distinguished between the two spectra in the region between 100 and $250 \mathrm{~cm}^{-1}$. The significance of this small difference will be discussed below where we show that the temperature broadening and evolution of the Raman signal for GeTe is anomalous.

In Figure 3a, the ambient pressure Raman spectra of the three materials at $300 \mathrm{~K}$ are shown. GeSe and SnSe spectra are similar while GeTe is different, because of a different crystalline structure but more importantly because phonon linewidth is much bigger. In Figure 

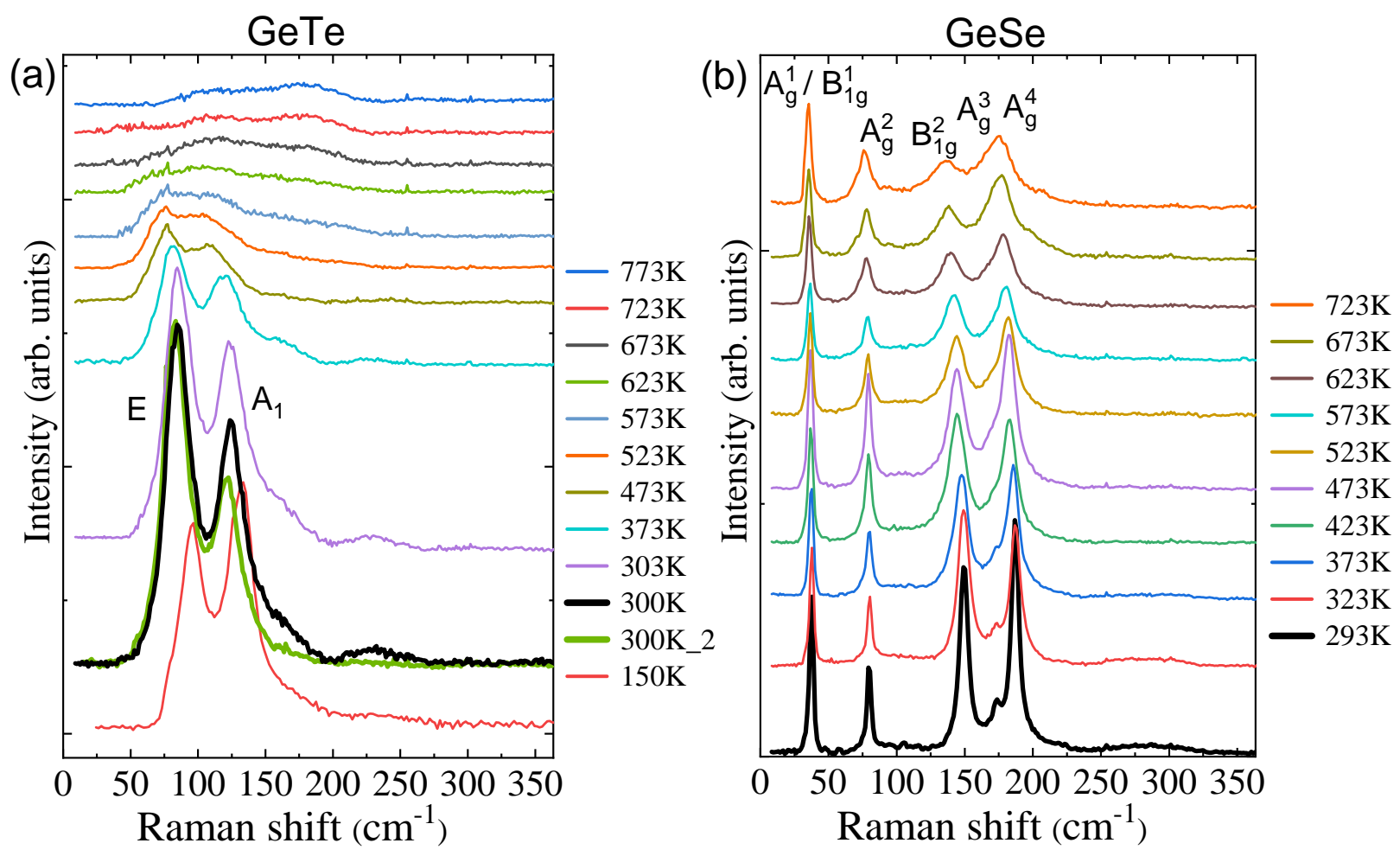

FIG. 2. Temperature dependent Raman spectra of (a) GeTe and (b) GeSe. The room temperature spectra are different because of the different crystal structure (rhombohedral for GeTe and orthorhombic for GeSe). The variation with temperature is also very different, with the anomalously broad peaks getting broader in GeTe and eventually disappearing in the high temperature Raman silent cubic phase. The $300 \mathrm{~K}$ spectra for GeTe correspond to the measurement before heating (black line) and after cooling down to room temperature (green line). In GeSe, the structure remains unchanged to the highest measured temperature, and peaks expectedly soften and broaden with temperature.

3b, the temperature variation of the GeTe Raman spectrum is compared to calculation. The simulation includes the frequency dependent two-phonon processes allowed by anharmonic phonon-phonon interaction [35] which is important in these materials, as well as thermal expansion. Other contributions to linewidth broadening, not included in the simulation, are electron-phonon scattering and scattering by lattice defects. In Figure $3 c$ the variation of the experimental and simulated linewidths is shown. The simulation reveals that at low temperature the anharmonic contribution is small as expected. The measured linewidths are significantly broader than the simulated ones and independent of temperature below 

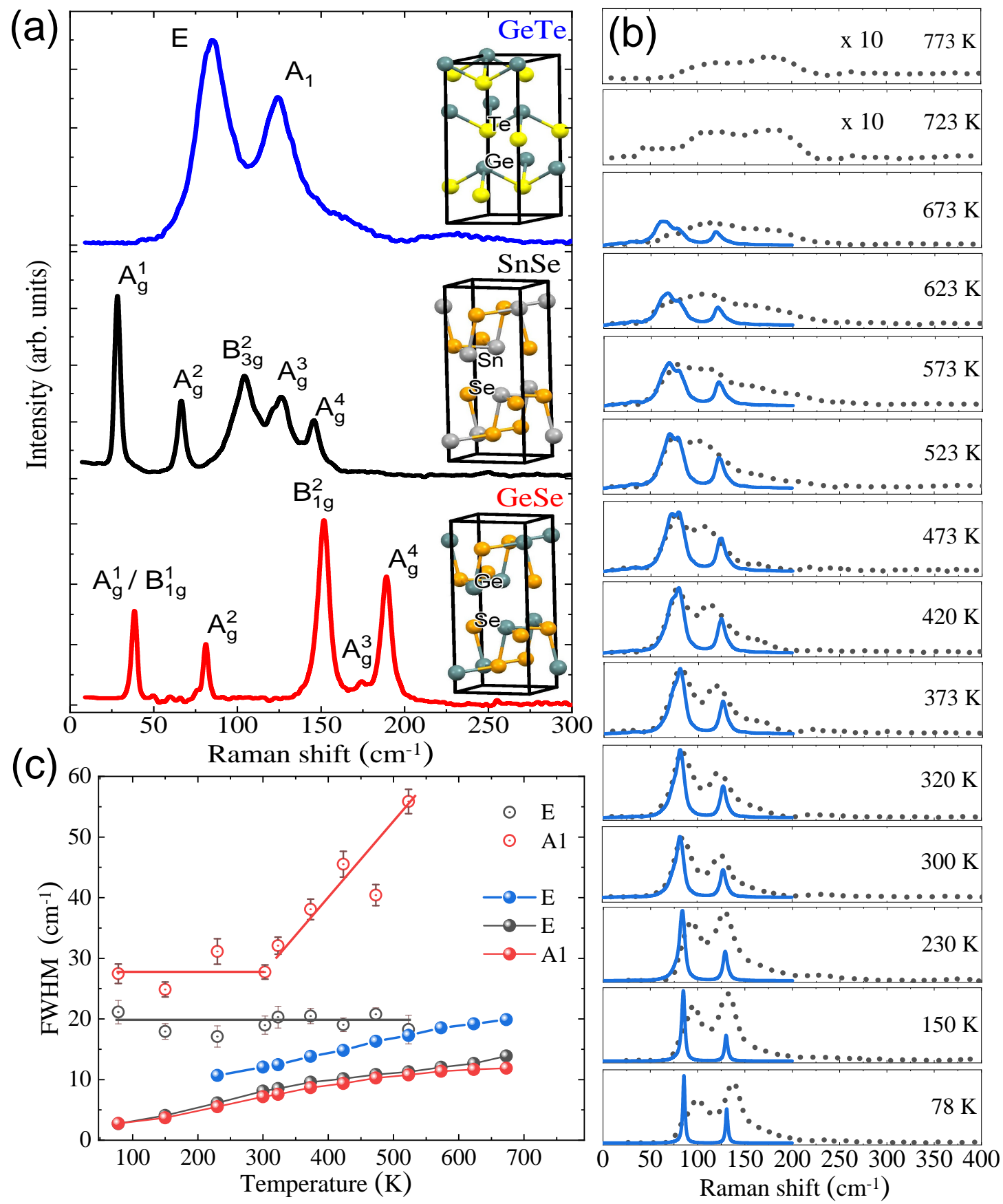

FIG. 3. (a) Ambient pressure, room temperature Raman spectra for GeSe, SnSe and GeTe, with the ambient condition rhombohedric R3m unit cell shown for GeTe and the orthorhombic Pnma unit cell shown for GeSe and SnSe. (b) Temperature dependence of experimental GeTe Raman spectra (dotted line, measured count-rate) and a comparison with calculation including anharmonicity (solid line, normalized to experimental count-rate). (c) Extracted variation (full width at half maximum) with temperature of experimental (empty circles) and simulated (full circles) linewidths in (b). The doubly degenerate E mode splits with temperature in simulation. 
$300 \mathrm{~K}$, indicating that the other contributions to linewidth are important. Above $600 \mathrm{~K}$ the experiment corresponds to a low intensity background. Above $700 \mathrm{~K}$, where a transition to the Raman silent cubic phase takes place, the background shifts to higher energy. Even at the lowest temperatures a high energy shoulder exists between 150 and $200 \mathrm{~cm}^{-1}$ which is not accounted for in the calculations. This shoulder transforms to a major part of the background at high temperature and in the cubic phase.

A part of the broadening over the whole temperature range, and the low intensity, high energy background in the Raman silent cubic phase can be both traced to the high propensity of GeTe to form Ge vacancies, particularly in the cubic structure [46].

Metavalent bonding is characterized by a relative ease in bond breaking which facilitates vacancy formation[47]. Indeed calculations [48, 49] and experiments $[15,40]$ indicate that large scale Ge vacancies are very easily formed in GeTe and should contribute to phonon linewidth through scattering [45]. Edwards et al.[49] show that Ge vacancies have a formation energy which is a third of Te vacancies and they do not induce localized gap states but delocalized states at the top of the valence band, giving rise to p-type metallic conductivity. Screening from this charge softens phonons [44] and will also contribute to Raman linewidth broadening. Finally, Park et al. [50] have shown that local Te rich phases can exhibit a variety of modes with energies ranging between 120 and $170 \mathrm{~cm}^{-1}$, the range of the background signal in our high temperature Raman spectra. These modes originate in Te-Te bonds (induced by Ge vacancies) and $\mathrm{GeTe}_{4}$ edge sharing octahedra. A further point to be noted is that though we recover the Raman signal at $300 \mathrm{~K}$ after having cooled the sample down (Figure 2a), ensuring that the measured high temperature changes are not due to extrinsic effects, differences appear in the high energy region between 100 and $200 \mathrm{~cm}^{-1}$ which corresponds to the signal from defects. Ge vacancies and the accompanying doping can thus explain the temperature dependence and the anomalous broadening of the Raman spectra in GeTe which anharmonic effects alone cannot account for.

The peculiar properties of GeTe and other tellurides like SnTe or PbTe have been highlighted by a recent classification of their chemical bonding which sets them apart from GeSe or SnSe [5, 6] as incipient metals with a metavalent bonding mechanism different from that of ionic or covalent solids or of conventional metals. This classification can be important for material design and understanding if it can be validated by experiment. Our pressure and temperature dependent, high resolution, low frequency Raman scattering results are 
consistent with this classification because they confirm the similarity of GeSe and SnSe and the singularity of GeTe. Temperature dependent Raman scattering experiments and simulations show that the anomalous broadening of the Raman lines observed in GeTe cannot be explained by anharmonicity alone. It is a sign of the peculiar chemical bonding in this material and a defect prone structure, known to result in strong doping and resultant phonon damping. On the other hand pressure dependent Raman spectra show the close similarity between the covalently bonded solids SnSe and GeSe. They also reveal the intriguing possibility that at higher pressure, SnSe and GeSe evolve in the direction of incipient metals, with anharmonic phonons, higher conductivity and metavalent bonding. This work also helps establish the relevant phase diagrams of all these materials, many aspects of which needed clarification.

This work was granted HPC resources of IDRIS under GENCI Project 7320. A. P. acknowledges the Indo-French Centre for the Promotion of Advanced Research (CEFIPRA, grant no. 5608-2) for postdoctoral fellowship.

* These authors contributed equally

† abhay.shukla@sorbonne-universite.fr

[1] G. Lucovsky, and R. M. White, Phys. Rev. B. 8, 660 (1973).

[2] K. Shportko, S. Kremers, M. Woda, D. Lencer, J. Robertson, and M. Wuttig, Nat. Mater. 7, $653(2008)$.

[3] D. Lencer, M. Salinga, B. Grabowski, T. Hickel, J. Neugebauer, and M. Wuttig, Nat. Mater. 7, 972 (2008).

[4] S. Lee, K. Esfarjani, T. Luo, J. Zhou, Z. Tian, and G. Chen, Nat. Commun. 5, 3525 (2014).

[5] M. Wuttig, V. Deringer, X. Gonze, C. Bichara, and J. Raty, Adv. Mater. 30, 1803777 (2018).

[6] J. Raty, M. Schumacher, P. Golub, V. Deringer, C. Gatti, and M. Wuttig, Adv. Mater. 31, $1806280(2019)$.

[7] B. Huang, and J. Robertson, Phys. Rev. B. 81, 081204 (2010).

[8] B.J. Kooi, and M. Wuttig, Adv. Mater. 32, 1908302 (2020).

[9] A. Pawbake, C. Bellin, L. Paulatto, K. Béneut, J. Biscaras, C. Narayana, D. Late, and A. Shukla, Phys. Rev. Lett. 122, 145701 (2019). 
[10] C. W. Li, J. Hong, A. F. May, D. Bansal, S. Chi, T. Hong, G. Ehlers, and O. Delaire, Nat. Phys. 11, 1063-1069 (2015).

[11] H. Wiedemeier, P. A. Siemers, Z. Anorg. Allg. Chem. 411, 90-96 (1975).

[12] A. Onodera, I. Sakamoto, Y. Fujii, N. Mo, and S. Sugai, Phys. Rev. B 56, 7935 (1997).

[13] M. Xu, S. Jakobs, R. Mazzarello, J. Cho, Z. Yang, H. Hollermann, D. Shang, X. Miao, Z. Yu, and L. Wang, J. Phys. Chem. C 121, 25447-25454 (2017).

[14] L. Zhao, S. Lo, Y. Zhang, H. Sun, G. Tan, C. Uher, C. Wolverton, V. Dravid, and G. Kanatzidis, Nature 508, 373-377 (2014).

[15] T. Chattopadhyay, J. Pannetier, and H. G. Von Schnering, J. Phys. Chem. Solids 47, 879-885 (1986).

[16] I. Efthimiopoulos, M. Berg, A. Bande, L. Puskar, E. Ritter, W. Xu, A. Marcelli, M. Ortolani, M. Harms, and J. Müller, Phys. Chem. Chem. Phys. 21, 8663-8678 (2019).

[17] I. Loa, R. J. Husband, R. A. Downie, S. R. Popuri, and J. W. Bos, J. Phys.: Condens. Matter 27, 072202 (2015).

[18] X. Chen, P. Lu, X. Wang, Y. Zhou, C. An, Y. Zhou, C. Xian, H. Gao, Z. Guo, and C. Park, Phys. Rev. B 96, 165123 (2017).

[19] K. Nguyen-Cong, J. Gonzalez, B. Steele, and I. Oleynik, J. Phys. Chem. C 122, 18274-18281 (2018).

[20] H. Yu, W. Lao, L. Wang, K. Li, and Y. Chen, Phys. Rev. Lett. 118, 137002 (2017).

[21] J. Yan, F. Ke, C. Liu, L. Wang, Q. Wang, J. Zhang, G. Li, Y. Han, Y. Ma, and C. Gao, Phys. Chem. Chem. Phys. 18, 5012-5018 (2016).

[22] J. C. Chervin, B. Canny, J. M. Besson, and P. Pruzan, Rev. Sci. Instrum. 66, 2595-2598 (1995).

[23] B. Couzinet, N. Dahan, G. Hamel, and J. Chervin, High Pressure Res. 23, 409-415 (2003).

[24] J. C. Chervin, B. Canny, and M. Mancinelli, Int. J. High Pressure Res. 21, 305-314 (2001).

[25] J. D. Barnett, S. Block, and G. J. Piermarini, Rev. Sci. Instrum. 44, 1-9 (1973).

[26] P. Giannozzi et al., J. Phys.: Condens. Matter 29, 465901 (2017).

[27] P. Giannozzi et al., J. Phys.: Condens. Matter 21, 395502 (2009).

[28] S. Baroni, S. De Gironcoli,A. Dal Corso, and P. Giannozzi, Rev. Mod. Phys. 73, 515 (2001).

[29] M. Lazzeri, and F. Mauri, Phys. Rev. Lett. 90, 036401 (2003).

[30] L. Paulatto, F. Mauri, and M. Lazzeri, Phys. Rev. B. 87, 214303 (2013). 
[31] D. R. Hamann, Phys. Rev. B. 88, 085117 (2013).

[32] M. Schlipf, and F. Gygi, Comput. Phys. Commun. 196, 36-44 (2015).

[33] J. P. Perdew, K. Burke, and M. Ernzerhof, Phys. Rev. Lett. 77, 3865 (1996).

[34] S. Grimme, J. Comput. Chem. 27, 1787-1799 (2006).

[35] L. Paulatto, I. Errea, M. Calandra, and F. Mauri, Phys. Rev. B. 91, 054304 (2015).

[36] R. A. Cowley, Rep. Prog. Phys. 31, 123 (1968).

[37] A. Okazaki, J. Phys. Soc. Jpn. 13, 1151-1155 (1958).

[38] H. R. Chandrasekhar, R. G. Humphreys, U. Zwick, and M. Cardona, Phys. Rev. B 15, 2177 (1977).

[39] H. B. Ribeiro, S. Ramos, L. Seixas, C. Matos, and M. Pimenta, Phys. Rev. B 100, 094301 (2019).

[40] F. Liu, P. Parajuli, R. Rao, P. Wei, A. Karunarathne, S. Bhattacharya, R. Podila, J. He, B. Maruyama, and G. Priyadarshan, Phys. Rev. B 98, 224309 (2018).

[41] Y. Kim, and I. Choi, J. Korean Phys. Soc. 72, 238-242 (2018).

[42] F. Cerdeira, T. A. Fjeldly, and M. Cardona, Phys. Rev. B. 8, 4734 (1973).

[43] D. Kumar, M. Chandrana, and M. S. Ramachandra Rao, Appl. Phys. Lett. 110, 191602 (2017).

[44] R. Shaltaf, X. Gonze, M. Cardona, R. K. Kremer, and G. Siegle, Phys. Rev. B. 79, 075204 (2009).

[45] D. Campi, L. Paulatto, G. Fugallo, F. Mauri, and M. Bernasconi, Phys. Rev. B. 95, 024311 (2017).

[46] M. Sist, H. Kasai, E. M. J. Hedegaard, and B. B. Iversen, Phys. Rev. B 97, 094116 (2018).

[47] M. Zhu, O. Cojocaru-Mirédin, A. Mio, J. Keutgen, M. Küpers, Y. Yu, J. Cho, R. Dronskowski, and M. Wuttig, Adv. Mater. 30, 1706735 (2018).

[48] W. Wełnic, A. Pamungkas, R. Detemple, C. Steimer, S. Blügel, and M. Wuttig, Nat. Mater. 5, 56-62 (2006).

[49] A. H. Edwards, A. C. Pineda, P. A. Schultz, M. G. Martin, A. P. Thompson, H. P. Hjalmarson, and C. J. Umrigar, Phys. Rev. B 73, 045210 (2006).

[50] J. Park, M. Song, S. Yoon, H. Lim, D. Jeong, C. Cheong, and H. Lee, Phys. Status Solidi A 210, 267-275 (2013). 